\title{
HEIGHT ZETA FUNCTIONS OF TWISTED PRODUCTS
}

\author{
Matthias Strauch AND Yuri Tschinkel
}

ABstract. We investigate analytic properties of height zeta functions of toric bundles over flag varieties.

\section{Introduction}

Let $X$ be a nonsingular algebraic variety over a number field $F$. We shall denote by $X(F)$ the set of $F$-rational points of $X$. Let $\mathcal{L}=\left(L,\left(\|\cdot\|_{v}\right)\right)$ be a metrized line bundle on $X$, i.e., a line bundle $L$ together with a family of $v$-adic metrics, where $v$ runs over the set $\operatorname{Val}(F)$ of normalized valuations of $F$. A metrized line bundle $\mathcal{L}$ defines a height function

$$
H_{\mathcal{L}}: X(F) \rightarrow \mathbb{R}_{>0}
$$

For appropriate subvarieties $U \subset X$ and line bundles $L$ we have

$$
N(U, \mathcal{L}, B):=\#\left\{x \in U(F) \mid H_{\mathcal{L}}(x) \leq B\right\}<\infty
$$

for all $B \in \mathbb{R}_{>0}$ (e.g., this holds for any $U \subset X$ if $L$ is ample). We are interested in the asymptotic behavior of this counting function as $B \rightarrow \infty$. It is expected that the behavior of such asymptotics can be described in geometrical terms $[7,1]$.

Definition 1.1. Let us denote by $\operatorname{Pic}(X)$ the Picard group of $X, \operatorname{Pic}(X)_{\mathbb{R}}=$ $\operatorname{Pic}(X) \otimes \mathbb{R}$. The cone of effective divisors of $X$ is the closed cone $\Lambda_{\text {eff }}(X) \subset$ $\operatorname{Pic}(X)_{\mathbb{R}}$ generated by the classes of effective divisors.

Let $[L] \in \operatorname{Pic}(X)$ be the class of $L$. Denote by $K_{X}$ the canonical line bundle on $X$.

Definition 1.2. Let $L$ be a line bundle on $X$ such that $[L]$ is contained in the interior of the cone $\Lambda_{e f f}(X)$. Define

$$
a(L):=\inf \left\{a \in \mathbb{R} \mid a[L]+\left[K_{X}\right] \in \Lambda_{\text {eff }}(X)\right\} .
$$

Assume that $\Lambda_{\text {eff }}(X)$ is a finitely generated polyhedral cone. Define $b(L)$ as the codimension of the minimal face of $\Lambda_{e f f}(X)$ which contains $a(L)[L]+\left[K_{X}\right]$.

Received January 13, 1997.

The first author was supported by the Graduiertenkolleg of the Mathematics Institute of the University of Bonn. 
In this paper we will consider the following class of varieties. Let $X$ be a smooth projective algebraic variety over $F$ with an action of a split algebraic torus $T$ and let $G$ be a semisimple split linear algebraic group over $F$. Denote by $P$ an $F$-rational parabolic subgroup of $G$. Assume that we have a morphism $\eta: P \rightarrow T$, it will define an action of $P$ on $X \times G$ by $(x, g) p \rightarrow\left(x \eta(p), p^{-1} g\right)$. There exists a smooth projective quotient of $X \times G$ by $P$ which we denote by $Y=Y_{\eta}:=X \times{ }^{P} G$. This quotient is a locally trivial bundle over $W=P \backslash G$ with fiber $X$.

For example, let us choose a Borel subgroup $B \subset G$ containing the maximal unipotent subgroup $U \subset B$, set $T=B / U$ and let $\eta$ be the canonical projection. Applying this construction to some smooth equivariant compactification of $T$ we will obtain an equivariant compactification of the homogeneous space $G / U$. Such compactifications are called horospherical varieties, see $[8,6]$ for a classification and a detailed study of their geometric properties over algebraically closed fields.

Definition 1.3. Let $U \subset X$ be a locally closed subset. We define the height zeta function by the series

$$
Z(U, \mathcal{L}, s):=\sum_{x \in U(F)} H_{\mathcal{L}}(x)^{-s}
$$

For appropriate $U$ and $L$ the series converges for $\operatorname{Re}(s) \gg 0$.

A tauberian theorem relates its analytic properties with the asymptotic properties of the counting function $N(U, \mathcal{L}, B)$. Analytic properties of height zeta functions are understood for flag varieties [7] and for toric varieties $[2,3,4]$.

Theorem 1.4. Let $X$ be a smooth projective toric variety over a number field $F$, $Y=X \times{ }^{P} G$ a twisted product as described above. Denote by $Y^{\circ}:=T \times{ }^{P} G \subset Y$ the Zariski open subset, obtained as a twisted product of $T$ with $P \backslash G$. Let $\mathcal{L}$ be a metrized line bundle on $Y$ (with the metrization introduced below), such that its class $[L]$ is contained in the interior of the cone of effective divisors. Then there exists a $\delta(\mathcal{L})>0$ such that the height zeta function has the following representation:

$$
Z\left(Y^{\circ}, \mathcal{L}, s\right)=\frac{\Theta(\mathcal{L})}{(s-a(L))^{b(L)}}+\frac{h(s)}{(s-a(L))^{b(L)-1}},
$$

where $a(L) \in \mathbb{Q}_{>0}, h(s)$ is a function which is holomorphic for $\operatorname{Re}(s) \geq a(L)$ $\delta(\mathcal{L})$ and $\Theta(\mathcal{L}) \neq 0$.

Theorem 1.5. For rational points on $Y^{\circ} \subset Y$ we have the following asymptotic formula

$$
N\left(Y^{\circ}, \mathcal{L}, B\right)=\frac{\Theta(\mathcal{L})}{a(L)(b(L)-1) !} B^{a(L)}(\log B)^{b(L)-1}(1+o(1))
$$


for $B \rightarrow \infty$, where $\Theta(\mathcal{L}) \neq 0$. Moreover,

$$
\Theta\left(-\mathcal{K}_{Y}\right)=\alpha(Y) \tau\left(\mathcal{K}_{Y}\right)
$$

where $\alpha(Y) \in \mathbb{Q}$ is a constant related to the geometry of the cone of effective divisors and $\tau\left(\mathcal{K}_{Y}\right)$ is the Tamagawa number defined by Peyre [9].

This proves the conjecture of Batyrev and Manin [1] about the distribution of rational points of bounded height for this class of varieties and the conjecture of Peyre about the asymptotic constant [9] (with respect to a specific metrization). As special cases it includes the results for flag varieties and for toric varieties obtained in $[7,1,4]$. Actually, it is not necessary to assume that $T$ and $G$ are split over $F$. In this paper we present the relevant constructions and give an outline of the argument. The proofs will appear in [12].

Similar results hold for twisted products of flag varieties with flag varieties. However, the proofs require certain restrictions on the twist $\eta$ [11]. An example which doesn't fit the picture given by the conjecture is contained in [5].

\section{Geometry}

Let $G$ be a split semisimple algebraic group defined over a number field $F$ and $P_{0}$ a minimal parabolic subgroup of $G$. Let $P$ be a standard parabolic subgroup, $X^{*}(P)$ the group of $F$-rational characters of $P$. We denote by $W=$ $P \backslash G$ the generalized flag variety. There is a monomorphism with finite cokernel $X^{*}(P) \rightarrow \operatorname{Pic}(W)$. We introduce coordinates in $X^{*}(P)_{\mathbb{C}}$ as follows. Let $\Delta_{0}$ be the set of simple roots determined by the maximal torus $T_{0}$ in the center of a Levi component of $P_{0}$. Let $\check{a}_{0}^{+} \subset X^{*}\left(P_{0}\right)$ be the closure of the positive Weyl chamber determined by $P_{0}$. There is an embedding of $X^{*}(P) \hookrightarrow X^{*}\left(P_{0}\right)$. Let $\check{a}_{P}^{+}$ be the closed cone $\check{a}_{0}^{+} \cap X^{*}(P)_{\mathbb{R}}$ which is spanned by a canonical basis $\omega_{1}, \ldots, \omega_{r}$. We identify $\mathbf{s}^{\prime}=\left(s_{1}, \ldots, s_{r}\right) \in \mathbb{C}^{r}$ with $s_{1} \omega_{1}+\cdots+s_{r} \omega_{r} \in X^{*}(P)_{\mathbb{C}}$.

Let $X$ be a variety with an action $\tau: X \times T \rightarrow X$ of a split algebraic torus $T$ and $\eta$ a morphism $P \rightarrow T$. Denote by $\pi_{X}: X \times T \rightarrow X$ the canonical projection. The twisted product $Y:=X \times{ }^{P} G$ has the following properties: We have a canonical morphism $\pi: Y \rightarrow W$ under which $Y$ is a locally trivial fiber bundle over $W$ with fiber $X$. Therefore, if $X$ is non-singular, so is $Y$. Let $L$ be a line bundle on $X$ and suppose that $\psi: \tau^{*} L \rightarrow \pi_{X}^{*} L$ is a $T$-linearization of $L$. Using this $T$-linearization one gets a canonical line bundle $L^{Y}$ on $Y$. Note that this line bundle and even its isomorphism class in $\operatorname{Pic}(Y)$ depend on the choice of the $T$-linearization. In fact, we get a homomorphism

$$
\operatorname{Pic}^{T}(X) \rightarrow \operatorname{Pic}(Y)
$$

from the group of isomorphism classes of $T$-linearized line bundles on $X$ to the Picard group of $Y$. One can show that if $X$ is non-singular there is an exact sequence

$$
0 \rightarrow \operatorname{Pic}(W) \stackrel{\pi^{*}}{\longrightarrow} \operatorname{Pic}(Y) \rightarrow \operatorname{Pic}(X) \rightarrow 0
$$


Now let us consider the case when $X$ is a toric variety. Let $T$ be a $d$ dimensional split algebraic torus over $F$. Let $M$ be the lattice of $F$-rational characters of $T$ and $N$ the dual lattice. Smooth equivariant compactifications of algebraic tori are described by combinatorial objects - complete regular $d$ dimensional fans $\Sigma \subset N \otimes \mathbb{R}$ (see [2]). Let $\Sigma(1):=\left\{e_{1}, \ldots, e_{n}\right\}$ be the set of 1-dimensional generators of $\Sigma$. Denote by $P L(\Sigma)$ the lattice of piecewise linear integral functions on $N$. Clearly, we have an embedding $M \hookrightarrow P L(\Sigma)$. An integral piecewise linear function $\varphi \in P L(\Sigma)$ is determined by the vector $\mathbf{s}=\left(s_{1}, \ldots, s_{n}\right)$, where $s_{i} \in \mathbb{Z}$ is the value of $\varphi$ on the generator of some 1 dimensional cone in $\Sigma(1),(i=1, \ldots, n)$. It will be convenient for us to work with these coordinates which we can assume to be complex. We write $\varphi_{\mathbf{s}}$ for the complex valued piecewise linear function thus obtained.

Let $\eta: P \rightarrow T$ be a morphism and $Y=Y_{\eta}:=X \times_{P} G$ the twisted product. We have a homomorphism $\check{\eta}: M \rightarrow X^{*}(P)$.

Proposition 2.1. 1. There is an exact sequence

$$
0 \rightarrow M \rightarrow P L(\Sigma) \oplus X^{*}(P) \rightarrow \operatorname{Pic}(Y) \rightarrow 0 .
$$

2. The cone of effective divisors $\Lambda_{\text {eff }}(Y)$ is the image of the simplicial cone $P L(\Sigma)^{+} \oplus \check{a}_{P}^{+}$, where $P L(\Sigma)^{+} \subset P L(\Sigma)_{\mathbb{R}}$ is the cone of piecewise linear functions $\varphi_{\mathbf{s}}$ such that $\varphi_{\mathbf{s}}\left(e_{j}\right) \geq 0$ for all $j=1, \ldots, n$.

3. The anticanonical class is given by the class of $\left(\varphi_{\Sigma}, 2 \rho_{P},\right)$, where $\varphi_{\Sigma}\left(e_{j}\right)=$ 1 for all $j=1, \ldots, n$ and $2 \rho_{P}$ is the sum of roots occuring in the unipotent radical of $P$ (counted with multiplicities).

One can find a description of these geometric properties for spherical varieties over algebraically closed fields in [6].

\section{Metrizations of line bundles}

Let $X$ be a smooth projective variety over $F$ and let $L$ be a line bundle on $X$.

Definition 3.1. Let $v$ be a place of $F$. A $v$-adic metric on $L$ is a family $\left(\|\cdot\|_{x}\right)_{x \in X\left(F_{v}\right)}$ of $v$-adic norms

$$
\|\cdot\|_{x}: x^{*} L \rightarrow \mathbb{R}
$$

such that for any open subset $U \subset X$ and any $s \in \Gamma(U, L)$ the map

$$
U\left(F_{v}\right) \rightarrow \mathbb{R}, x \mapsto\left\|x^{*} s\right\|_{x},
$$

is continuous, where $U\left(F_{v}\right)$ is given the $v$-adic topology.

We will write $\left(\|\cdot\|_{x}\right)$ instead of $\left(\|\cdot\|_{x}\right)_{x \in X\left(F_{v}\right)}$ if the place $v$ is clear from the context. In the sequel we will use the symbol $\|\cdot\|_{v}$ to denote a $v$-adic metric on $L$. 
The compactness of $X\left(F_{v}\right)$ implies that any two $v$-adic metrics $\|\cdot\|_{v}$ and $\|\cdot\|_{v}^{\prime}$ on $L$ are equivalent in the sense that there exist constants $c_{2} \geq c_{1}>0$ such that for all $x \in X\left(F_{v}\right)$ and all $s \in x^{*} L$ we have [10]

$$
c_{1}\|s\|_{x} \leq\|s\|_{x}^{\prime} \leq c_{2}\|s\|_{x}
$$

Given a $v$-adic metric $\|\cdot\|_{v}$ on $L$ we get a $v$-adic metric $\|\cdot\|_{v}^{\vee}$ on the dual line bundle $L^{\vee}$ by setting

$$
\|t\|_{x}^{\vee}:=\frac{|t(s)|_{v}}{\|s\|_{x}}
$$

for arbitrary $s \in x^{*} L-\{0\}$. If we are given a $v$-adic metric $\|\cdot\|_{v}^{\prime}$ on another line bundle $L^{\prime}$ then we get a canonical $v$-adic metric $\|\cdot\|_{v}\|\cdot\|_{v}^{\prime}$ on $L \otimes L^{\prime}$.

Suppose that $L$ is generated by global sections, and let $\left(s_{j}\right)_{j=0, \ldots, q}$ be a family of $F$-rational global sections which generate the space $\Gamma(X, L)$. Let $v \in \operatorname{Val}(F), x \in X\left(F_{v}\right)$ and $s \in x^{*} L-\{0\}$. We put

$$
\|s\|_{x}:=\left(\max _{0 \leq j \leq q}\left|\frac{s_{j}}{s}(x)\right|_{v}\right)^{-1}
$$

and $\|0\|_{x}=0$. The family $\left(\|\cdot\|_{x}\right)_{x \in X\left(F_{v}\right)}$ is then a $v$-adic metric on $L$, called the $v$-adic metric associated with the family $\left(s_{j}\right)_{j=0, \ldots, q}$ of global sections of $L$.

Definition 3.2. An adelic metric on $L$ is a family $\left(\|\cdot\|_{v}\right)_{v \in \operatorname{Val}(F)}$ of $v$-adic metrics $\|\cdot\|_{v}$ on $L$ such that the following holds: there are line bundles $L_{1}, L_{2}$ on $X$ which are generated by global sections, families $\left(s_{j}^{(1)}\right)_{j=0, \ldots, q_{1}}$ (resp. $\left(s_{j}^{(2)}\right)_{j=0, \ldots, q_{2}}$ ) of global sections of $L_{1}$ (resp. $L_{2}$ ) generating $\Gamma\left(X, L_{1}\right)$ (resp. $\left.\Gamma\left(X, L_{2}\right)\right)$ and an isomorphism $L \rightarrow L_{1} \otimes L_{2}^{\vee}$ such that for almost all $v$ the norm $\|\cdot\|_{v}$ is the pullback of $\|\cdot\|_{v}^{(1)} \cdot\left(\|\cdot\|_{v}^{(2)}\right)^{\vee}$ under this isomorphism, where $\|\cdot\|_{v}^{(1)}$ (resp. $\|\cdot\|_{v}^{(2)}$ ) is the $v$-adic metric associated to $\left(s_{j}^{(1)}\right)_{j=0, \ldots, q_{1}}$ (resp. $\left.\left(s_{j}^{(2)}\right)_{j=0, \ldots, q_{2}}\right)$.

In the sequel we will write $\left(\|\cdot\|_{v}\right)$ for an adelic metric $\left(\|\cdot\|_{v}\right)_{v \in \operatorname{Val}(F)}$. A pair $\mathcal{L}=\left(L,\left(\|\cdot\|_{v}\right)\right)$ consisting of a line bundle $L$ on $X$ and an adelic metric $\left(\|\cdot\|_{v}\right)$ on $L$ will be called a metrized line bundle or a metrization of $L$.

Definition 3.3. Let $\mathcal{L}$ be a metrized line bundle on $X$. It defines the height function $H_{\mathcal{L}}: X(F) \rightarrow \mathbb{R}_{>0}$ by

$$
H_{\mathcal{L}}(x)=\prod_{v \in \operatorname{Val}(F)}\left\|x_{v}^{*} s\right\|_{x_{v}}^{-1}
$$

where $x_{v}$ is the image of $x$ under the embedding $X(F) \rightarrow X\left(F_{v}\right)$ and $s$ is any $F$-rational local section of $L$ at $x$ with $s(x) \neq 0$. 
It is easy to show that for a given $x \in X(F)$ almost all factors in the product equal 1. By the product formula, the height is independent of the choice of an $F$-rational section $s$.

Let $Y$ be a twisted product of a flag variety $W$ with a smooth projective variety $X$ equipped with an action of a split algebraic torus $T$. Let $L$ be a line bundle on $X$ and $\psi: \tau^{*} L \rightarrow \pi_{X}^{*} L$ its $T$-linearization. Suppose that we are given a $v$-adic metric $\|\cdot\|_{v}$ on $L$. Let $t \in T\left(F_{v}\right)$. For any $x \in X\left(F_{v}\right)$ we have an induced isomorphism

$$
\psi_{x, t}: x^{*} L=\left(x t, t^{-1}\right)^{*}\left(\tau^{*} L\right) \stackrel{\sim}{\longrightarrow}\left(x t, t^{-1}\right)^{*}\left(\pi_{X}^{*} L\right)=(x t)^{*} L .
$$

This allows to define $\|\cdot\|_{x, t}: x^{*} L \rightarrow \mathbb{R}$ by $\|s\|_{x, t}:=\left\|\psi_{x, t}(s)\right\|_{x t}$. It is easy to check that $\|\cdot\|_{v, t}:=\left(\|\cdot\|_{x, t}\right)_{x \in X\left(F_{v}\right)}$ is a new $v$-adic metric on $L$.

Denote by $\mathbb{K}_{T, v} \subset T\left(F_{v}\right)$ the maximal compact subgroup of $T\left(F_{v}\right)$. We shall call the $v$-adic metric $\|\cdot\|_{v}$ on $L$ admissible if for all $t \in \mathbb{K}_{T, v}$ we have $\|\cdot\|_{v, t}=\|\cdot\|_{v}$. This does not depend on $\psi$. An adelic metric $\left(\|\cdot\|_{v}\right)_{v \in \operatorname{Val}(F)}$ is called admissible if for all $v \in \operatorname{Val}(F)$ the $v$-adic metric $\|\cdot\|_{v}$ is admissible. It is clear that if $t=\left(t_{v}\right)_{v} \in T\left(\mathbb{A}_{F}\right)$ and $\left(\|\cdot\|_{v}\right)$ is an admissible adelic metric on $L$ then $\left(\|\cdot\|_{v, t_{v}}\right)_{v \in \operatorname{Val}(F)}$ is an adelic metric on $L$. We will denote this metrization of $L$ by $\mathcal{L}(t)$.

Let $\mathbb{K}_{G}=\prod_{v \in \operatorname{Val}(F)} \mathbb{K}_{G, v}$ be a maximal compact subgroup of $G\left(\mathbb{A}_{F}\right)$ such that we have the Iwasawa decomposition $G\left(\mathbb{A}_{F}\right)=P_{0}\left(\mathbb{A}_{F}\right) \mathbb{K}_{G}$. Let $(x, g) \in$ $X\left(F_{v}\right) \times G\left(F_{v}\right)$ be an element in the preimage of $y \in Y\left(F_{v}\right)$ under the projection $\pi_{Y}: X \times G \rightarrow Y$. Then, by the construction of $L^{Y}$ via $\psi: \tau^{*} L \rightarrow \pi_{X}^{*} L$, there is a canonical isomorphism

$$
\zeta_{x, g}: y^{*} L^{Y} \rightarrow x^{*} L
$$

Let $g=p k, p \in P\left(F_{v}\right), k \in \mathbb{K}_{G, v}$. Then we define a $v$-adic norm

$$
\|\cdot\|_{v}: y^{*} L^{Y} \rightarrow \mathbb{R}
$$

by

$$
\|s\|_{y}:=\left\|\zeta_{x, g}(s)\right\|_{x, \eta(p)} .
$$

This is well defined and gives a $v$-adic metric $\|\cdot\|_{v}^{Y}$ on $L^{Y}$. If $\left(\|\cdot\|_{v}\right)$ is an admissible adelic metric on $L$, then $\left(\|\cdot\|_{v}^{Y}\right)$ is an adelic metric on $L^{Y}$. We put $\mathcal{L}^{Y}:=\left(L^{Y},\left(\|\cdot\|_{v}^{Y}\right)\right)$.

Let $(x, g) \in \pi_{Y}^{-1}(y)$ for $y \in Y(F), g=p k, p \in P\left(\mathbb{A}_{F}\right), k \in \mathbb{K}_{G}$. By the construction above, we have

$$
H_{\mathcal{L}^{Y}}(y)=H_{\mathcal{L}(\eta(p))}(x) .
$$

Therefore, we get a formal expression for the height zeta function of the twisted product $Y=X \times{ }^{P} G$ corresponding to a metrized line bundle $\mathcal{L}^{Y} \otimes \pi^{*} \mathcal{L}_{W}$, where $\mathcal{L}$ is a metrized line bundle on $X$, consisting of a $T$-linearized line bundle $L$ and 
an admissible adelic metric, and $\mathcal{L}_{W}$ is a metrized line bundle on $W=P \backslash G$. Using the relation

$$
H_{\mathcal{L}^{Y} \otimes \pi^{*} \mathcal{L}_{W}}(y)=H_{\mathcal{L}^{Y}}(x) H_{\mathcal{L}_{W}}(\pi(y))
$$

we have formally

$$
\begin{aligned}
Z_{Y}\left(\mathcal{L}^{Y} \otimes \mathcal{L}_{W}, s\right) & =\sum_{\gamma \in P(F) \backslash G(F)} H_{\mathcal{L}_{W}}(\gamma)^{-s} \sum_{y \in \pi^{-1}(\gamma)} H_{\mathcal{L}^{Y}}(y)^{-s} \\
& =\sum_{\gamma \in P(F) \backslash G(F)} H_{\mathcal{L}_{W}}(\gamma)^{-s} \sum_{x \in X(F)} H_{\mathcal{L}\left(\eta\left(p_{\gamma}\right)\right)}(x)^{-s} \\
& =\sum_{\gamma \in P(F) \backslash G(F)} H_{\mathcal{L}_{W}}(\gamma)^{-s} Z_{X}\left(\mathcal{L}\left(\eta\left(p_{\gamma}\right)\right), s\right),
\end{aligned}
$$

where $\gamma=p_{\gamma} k_{\gamma}, p_{\gamma} \in P\left(\mathbb{A}_{F}\right), k_{\gamma} \in \mathbb{K}_{G}$.

Our next goal is to describe explicit metrizations of line bundles on twisted products of toric varieties with generalized flag varieties.

We have the logarithmic map

$$
T\left(F_{v}\right) / \mathbb{K}_{T, v} \rightarrow N
$$

(resp. $N \otimes \mathbb{R}$ for archimedean $v$ ) and we denote by $\bar{t}_{v}$ the image of $t_{v} \in N$. For every $g_{v}=\in G\left(F_{v}\right)$ we choose a representative in $P\left(F_{v}\right)$ which we will denote by $p\left(g_{v}\right)$. For every $g_{v} \in G\left(F_{v}\right)$ we define a homomorphism $H_{P, v}\left(g_{v}\right): X^{*}(P)_{\mathbb{C}} \rightarrow$ $\mathbb{C}$ by

$$
H_{P, v}\left(g_{v}, \lambda\right)=<\lambda, H_{P, v}\left(g_{v}\right)>=\log \left(\left|\lambda\left(p\left(g_{v}\right)\right)\right|_{v}\right)
$$

for $\lambda \in X^{*}(P)$ and extend this by linearity.

Definition 3.4. We define a pairing

$$
H_{\Sigma, v}: T\left(F_{v}\right) \times P L(\Sigma)_{\mathbb{C}} \rightarrow \mathbb{C}, \quad H_{\Sigma, v}\left(t_{v}, s\right)=e^{\varphi_{\mathbf{s}}\left(\bar{t}_{v}\right) \log q_{v}}
$$

where $q_{v}$ equals the number of elements in the residue field of $F_{v}$ for nonarchimedean $v$ and $\log q_{v}=1$ for archimedean $v$. Denote by $H_{\Sigma}:=$ $\prod_{v \in \operatorname{Val}(F)} H_{\Sigma, v}$. We define

$$
H_{W, v}: G\left(F_{v}\right) \times X^{*}(P)_{\mathbb{C}} \rightarrow \mathbb{C}, \quad H_{W, v}\left(g_{v}, s^{\prime}\right)=e^{<s^{\prime}, H_{P, v}\left(p\left(g_{v}\right)\right)>},
$$

and let $H_{W}:=\prod_{v \in \operatorname{Val}(F)} H_{W, v}$ and $H_{P}:=\sum_{v \in \operatorname{Val}(F)} H_{P, v}$. We define a pairing

$$
\begin{aligned}
& H_{\Sigma, \eta, v}:\left(T\left(F_{v}\right) \times G\left(F_{v}\right)\right) \times\left(P L(\Sigma)_{\mathbb{C}} \times X^{*}(P)_{\mathbb{C}}\right) \rightarrow \mathbb{C}, \\
& H_{\Sigma, \eta, v}\left(\left(t_{v}, g_{v}\right),\left(s, s^{\prime}\right)\right)=H_{\Sigma, v}\left(t_{v} \eta\left(p\left(g_{v}\right)\right), s\right) \cdot H_{W, v}\left(g_{v}, s^{\prime}\right) .
\end{aligned}
$$

We have the pairing

$$
H_{\Sigma, \eta}=\prod_{v \in \operatorname{Val}(F)} H_{\Sigma, \eta, v}:\left(T\left(\mathbb{A}_{F}\right) \times G\left(\mathbb{A}_{F}\right)\right) \times\left(P L(\Sigma)_{\mathbb{C}} \times X^{*}(P)_{\mathbb{C}}\right) \rightarrow \mathbb{C} .
$$


Proposition 3.5. The pairing $H_{\Sigma, \eta}$ descends to a pairing

$$
H_{\Sigma, \eta}: Y^{\circ}(F) \times \operatorname{Pic}(Y)_{\mathbb{C}} \rightarrow \mathbb{C} .
$$

Let $L$ be a line bundle and $[L]$ its class in $\operatorname{Pic}(Y)$. Denote by $Y^{\circ}=T \times{ }^{P} G \subset Y$. Then the restriction of the above pairing to $Y^{\circ}(F) \times[L]$ gives a height function with respect to some metrization of $L$.

The proof of this proposition proceeds by comparing the metrization of line bundles on $Y$ given by this pairing with the general construction of metrizations of line bundles on twisted products described above.

\section{Height zeta functions}

For $\left(\mathbf{s}, \mathbf{s}^{\prime}\right) \in P L(\Sigma)_{\mathbb{C}} \oplus X^{*}(P)_{\mathbb{C}}$ we define a zeta function by the series

$$
Z_{\Sigma, \eta}\left(\mathbf{s}, \mathbf{s}^{\prime}\right)=\sum_{y \in Y^{\circ}(F)} H_{\Sigma, \eta}\left(y,\left(-\mathbf{s},-\mathbf{s}^{\prime}\right)\right) .
$$

This zeta function is the extension of the one-parameter height zeta function considered above to the whole complexified Picard group.

Proposition 4.1. The series $Z_{\Sigma, \eta}\left(\mathbf{s}, \mathbf{s}^{\prime}\right)$ converges absolutely and uniformly to a holomorphic function for $\operatorname{Re}(\mathbf{s}) \in \mathbb{R}_{>1}^{n}$ and $\operatorname{Re}\left(\mathbf{s}^{\prime}\right) \in \mathbb{R}_{>1}^{r}$. Moreover, it descends to a function on $\operatorname{Pic}(Y)_{\mathbb{C}}$ which is holomorphic in the interior of the cone $\Lambda_{\text {eff }}(Y)+\left[-K_{Y}\right]$.

Recall that $Y^{\circ} \rightarrow W=P \backslash G$ is a fiber bundle with fibers isomorphic to $T$. Using the absolute convergence we can rearrange the terms summing first over the rational points in the base of this fibration and then over the rational points in each fiber. We obtain

$$
Z_{\Sigma, \eta}\left(s, s^{\prime}\right)=\sum_{\gamma \in P(F) \backslash G(F)} H_{W}\left(\gamma,-s^{\prime}\right) \sum_{t \in T(F)} H_{\Sigma}\left(t \eta\left(p_{\gamma}\right),-s\right) .
$$

Using the Poisson formula on the group $T\left(\mathbb{A}_{F}\right)$ and the $\mathbf{K}_{T}$-invariance of the pairing $H_{\Sigma}$ we have [2]:

\section{Proposition 4.2.}

$$
Z_{\Sigma, \eta}\left(s, s^{\prime}\right)=\sum_{\gamma \in P(F) \backslash G(F)} e^{<-s^{\prime}, H_{P}(\gamma)>} \int_{\mathcal{A}^{*}} \hat{H}_{\Sigma}(\chi,-s) \chi^{-1}\left(\eta\left(p_{\gamma}\right)\right) d \chi
$$

where $\mathcal{A}^{*}$ is the group of topological characters of $T\left(\mathbb{A}_{F}\right)$ which are trivial on the

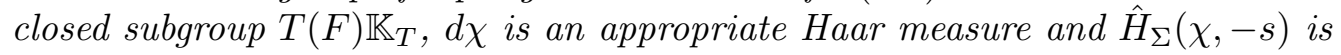
the Fourier transform of the function $H_{\Sigma}(x,-s)$ on $T\left(\mathbb{A}_{F}\right)$. 
The embedding $M \hookrightarrow P L(\Sigma)$ induces a map of tori $T_{P L} \rightarrow T$. We have $T_{P L}=\mathbf{G}_{m}^{n}$. A character $\chi \in \mathcal{A}^{*}$ defines characters $\left(\chi_{1}, \ldots, \chi_{n}\right)$ where $\chi_{j}$ are characters $\mathbf{G}_{m}\left(\mathbb{A}_{F}\right)$ invariant under the closed subgroup $\mathbf{G}_{m}(F) \mathbb{K}$, where $\mathbb{K} \subset$ $\mathbf{G}_{m}\left(\mathbb{A}_{F}\right)$ is the maximal compact subgroup. Using the explicit computation of $\hat{H}_{\Sigma}(\chi,-s)[2]$, and interchanging summation and integration we have

$$
\begin{aligned}
Z_{\Sigma, \eta}\left(\mathbf{s}, \mathbf{s}^{\prime}\right) & =\int_{\mathcal{A}^{*}} \prod_{j=1}^{n} L\left(s_{j}, \chi_{j}\right) \zeta_{\Sigma, \infty}(\mathbf{s}, \chi)\left(\sum_{\gamma \in P(F) \backslash G(F)} e^{<-s^{\prime}, H_{P}(\gamma)>} \chi^{-1}\left(\eta\left(p_{\gamma}\right)\right)\right) d \chi \\
& =\int_{\mathcal{A}^{*}} \prod_{j=1}^{n} L\left(s_{j}, \chi_{j}\right) E_{P}^{G}\left(s^{\prime}, \chi \circ \eta\right) \zeta_{\Sigma, \infty}(\mathbf{s}, \chi) d \chi
\end{aligned}
$$

Here we denoted by

$$
E_{P}^{G}\left(s^{\prime}, \chi \circ \eta\right):=\sum_{\gamma \in P(F) \backslash G(F)} e^{<-s^{\prime}, H_{P}(\gamma)>} \chi^{-1}\left(\eta\left(p_{\gamma}\right)\right)
$$

the Eisenstein series and by $L(s, \chi)$ the Hecke $L$-function.

The analytic continuation of this integral and the description of its poles uses uniform estimates of Hecke L-functions, Eisenstein series and $\zeta_{\Sigma, \infty}(\mathbf{s}, \chi)$. There is a canonical map $y: \mathcal{A}^{*} \rightarrow \sum_{v \mid \infty} M_{\mathbb{R}}$ with finite kernel. Let \|\|$_{\infty}$ be a norm on $\sum_{v \mid \infty} M_{\mathbb{R}}$ and \|\| a norm on $X^{*}(P)_{\mathbb{R}}$.

Proposition 4.3. Let $E_{P}^{G}\left(\mathbf{s}^{\prime}, \chi\right)$ be the Eisenstein series with unramified character $\chi \in\left(P\left(\mathbb{A}_{F}\right) / P(F)\right)^{*}$. For all $\varepsilon>0$ there exists a $\delta>0$ such that for all $\mathbf{s}^{\prime}$ with $\operatorname{Re}\left(\mathbf{s}^{\prime}\right)$ contained in any compact $K$ in the domain $0<\left|\operatorname{Re}\left(s_{j}\right)-1\right|<\delta$ for $j=1, \ldots, r$ and all $\chi \in\left(P\left(\mathbb{A}_{F}\right) / P(F)\right)^{*}$ there exists a constant $c(K, \varepsilon)$ depending only on $K$ and $\varepsilon$ such that

$$
\left|E_{P}^{G}\left(s^{\prime}, \chi\right)\right| \leq c(K, \varepsilon)\left(1+\left\|\operatorname{Im}\left(s^{\prime}\right)\right\|+\|y(\chi)\|_{\infty}\right)^{\varepsilon} .
$$

The proof of this proposition uses the Phragmen-Lindelöf principle and the theory of the constant term of Eisenstein series. The proof of our theorem 1.4 is similar to the proof of the main technical theorem in [4]. The proof of the theorem 1.5 combines the proofs of corresponding statements for toric varieties [3] and for generalized flag varieties [9].

\section{Acknowledgements}

We are very grateful to V. Batyrev and J. Franke for helpful discussions and collaboration on related questions. Part of this work was done while the second author was visiting ENS in Paris and MPI in Bonn. He would like to thank both institutions for ideal working conditions. 


\section{References}

1. V. Batyrev and Yu. I. Manin, Sur le nombre des points rationnels de hauteur bornée des variétés algébriques, Math. Ann., 286 (1990), 27-43.

2. V. Batyrev and Yu. Tschinkel, Rational points of bounded height on compactifications of anisotropic tori, Internat. Math. Res. Notices 12 (1995), 591-635.

3. __ Manin's conjecture for toric varieties, J. Alg. Geom. (to appear).

4. __ Height zeta functions of toric varieties, preprint, Ecole Norm. Sup., (1996).

5. _ Rational points on some Fano cubic bundles, C. R. Acad. Sci. Paris Ser. I 323 (1996), 41-46.

6. M. Brion, Variétés sphériques et théorie de Mori, Duke Math. J. 72 (1993), 369-404.

7. J. Franke, Yu. I. Manin, and Yu. Tschinkel, Rational points of bounded height on Fano varieties, Invent. Math. 95 (1989), 321-235.

8. F. Pauer, Normale Einbettungen von G/U, Math. Ann. 257 (1981), 371-396.

9. E. Peyre, Hauteurs et nombres de Tamagawa sur les variétés de Fano, Duke Math. J. 79 (1995), 101-218.

10. J. Silverman, The theory of height functions, in Arithmetic geometry, ed. G. Cornell, J. Silverman, Springer, New York, 1986.

11. M. Strauch, Rational points of bounded height on twisted products of generalized flag varieties, in preparation.

12. M. Strauch and Yu. Tschinkel, Height zeta functions of toric bundles over flag varieties, in preparation.

Mathematisches Institut, Universität Bonn, Beringstr. 1, 53117 Bonn, GeRMANY

E-mail address: mats@rhein.iam.uni-bonn.de

Dept. of Mathematics, U. I. C., Chicago, IL 60607-7045, U.S.A.

E-mail address: yuri@math.uic.edu 